\title{
Elementos precipitadores/intensificadores da violência conjugal em tempo da Covid-19
}

\author{
Marital violence precipitating/intensifying elements during the \\ Covid-19 pandemic
}

Andrey Ferreira da Silva (http://orcid.org/0000-0002-1038-7443) ${ }^{1}$

Fernanda Matheus Estrela (https://orcid.org/0000-0001-7501-6187) ${ }^{2}$

Caroline Fernandes Soares e Soares (https://orcid.org/0000-0003-4464-8389) ${ }^{2}$

Júlia Renata Fernandes de Magalhães (https://orcid.org/0000-0003-0631-2374) ${ }^{1}$

Nayara Silva Lima (https://orcid.org/0000-0001-7911-012X) ${ }^{3}$

Ariane Cedraz Morais (https://orcid.org/0000-0001-9445-4596) ${ }^{2}$

Nadirlene Pereira Gomes (https://orcid.org/0000-0002-6043-3997) ${ }^{1}$

Vera Lúcia de Azevedo Lima (https://orcid.org/0000-0003-0094-4530) ${ }^{4}$

${ }^{1}$ Escola de Enfermagem, Universidade Federal da

Bahia. Av. Dr. Augusto Viana s/n, Canela. 40110-060

Salvador BA Brasil.

silva.andrey1991@

hotmail.com

${ }^{2}$ Departamento de Saúde,

Universidade Estadual de

Feira de Santana. Feira de

Santana BA Brasil.

${ }^{3}$ Secretaria Municipal de

Saúde. Salvador BA Brasil.

${ }^{4}$ Universidade Federal do

Pará. Belém PA Brasil.

\begin{abstract}
The study aims to identify marital violence precipitating/intensifying elements during the COVID-19 pandemic. This is a narrative review of the literature, and the search was carried out in May 2020. We employed the PubCovid-19 platform, which is indexed in the United States National Library of Medicine (PubMed) and the Excerpta Medica (EMBASE) database. English descriptors "Domestic violence", "COVID-19", and "Intimate Partner Violence" were used in the search, and nine papers were selected for full-text reading. Three empirical categories were elaborated from the exploration of the selected material: Economic instability, Alcohol and other drugs usel abuse, and Weaker women's support network. Support networks for women in situations of marital violence should be expanded in this pandemic context, with emphasis on the use of digital technologies as possible tools for screening pandemi$c$-related violence cases.
\end{abstract}

Key words COVID-19, Pandemic, Intimate partner violence
Resumo $O$ estudo tem por objetivo identificar elementos precipitadores/intensificadores da violência conjugal em tempos de pandemia da Covid-19. Trata-se de uma revisão narrativa da literatura, tendo a busca sido realizada no mês de maio de 2020. Utilizou-se a plataforma PubCovid-19, a qual está indexada na Biblioteca Nacional de Medicina dos Estados Unidos (PubMed) $e$ no Banco de dados Excerpta Medica (EMBASE). Para a realização da busca foram utilizados os seguintes descritores em inglês: "Domestic violente"; "Covid-19" e "Intimate Partner Violence". Foram selecionados nove artigos para leitura na integra. A partir da exploração do material selecionado, foram elaboradas três categorias empíricas a saber: Instabilidade econômica, Uso/abuso de álcool e outras drogas e Enfraquecimento da rede de apoio da mulher. É importante que nesse contexto de pandemia, sejam ampliadas as redes de apoio à mulher em situação de violência conjugal, com destaque para o uso de tecnologias digitais como possíveis ferramentas para a triagem de casos de violência em tempos de pandemia.

Palavras-chave Covid-19, Pandemia, Violência por parceiro intimo 


\section{Introdução}

O distanciamento social, devido à pandemia da Covid-19, tem repercutido negativamente sobre a vida das mulheres, o que se expressa por meio dos crescentes índices de violência conjugal. Diante da preocupação mundial com esta exposição feminina, que gera adoecimentos e óbitos, e a necessidade de intervenção nesta conjuntura, urge o entendimento acerca dos fatores que precipitam e/ou intensificam tal fenômeno.

Contextualizando a pandemia do coronavírus (SARS-CoV-2), causador da Covid-19, este se tornou um desafio global para a saúde pública após rápida disseminação por todo o mundo, depois do primeiro caso oficial notificado em Wuhan, na China ${ }^{1}$. Em seguida à elevação drástica dos casos, em 30 de janeiro, a doença foi declarada como uma Emergência de Saúde Pública de importância internacional (ESPII) ${ }^{2}$, sendo essa responsável, até 11 de maio de 2020, por mais de 4 milhões de casos e 278 mil mortes distribuídos em todo o mundo ${ }^{1}$. No Brasil, a situação é extremamente preocupante, visto que, até o dia 14 de maio, ultrapassa 202 mil o número de casos confirmados e mais de 13 mil óbitos pela doença, tornando o $6^{\circ}$ país do mundo com mais casos ${ }^{3,4}$.

Diante o contexto internacional e nacional, fez-se necessária a adoção de medidas protetivas na tentativa de diminuir a morbimortalidade da doença. Assim, à medida que a doença atinge a fase de transmissão comunitária em diversas cidades brasileiras, várias ações foram elaboradas para controle da curva epidêmica ${ }^{5}$. Por se tratar de uma doença com elevado grau de transmissibilidade, o controle requer medidas de restrição de contato, distanciamento social e quarentena que, com base em experiências internacionais, são eficazes para a prevenção ${ }^{6}$. Este contexto, em que o ambiente doméstico tornou-se o lugar mais seguro para conter a transmissibilidade da COVID-19, vem trazendo desdobramentos em todas as esferas da sociedade, inclusive para muitas mulheres, visto que é justamente no espaço privado onde são vítimas de violência doméstica, de modo que ficar em casa não é sinônimo de proteção ${ }^{7}$.

Considerando a violência na conjugalidade, notícias divulgadas na mídia e relatórios de organizações internacionais sinalizam para aumentos significativos de casos nesse período de pandemia em todo o mundo. Na China e na Itália, os registros policiais de violência doméstica triplicaram e duplicaram durante a epidemia, respectivamente, quando comparadas ao mesmo período em 20198,9. Na França, que já possui uma das maiores taxas de violência da Europa, houve ainda um aumento de mais de 30\% após a implementação da quarentena domiciliar ${ }^{10}$. No Brasil, esse aumento foi de $18 \%$ nas denúncias aos serviços Disque 100 e Ligue 180, segundo a Ouvidoria Nacional dos Direitos Humanos (ONDH), do Ministério da Mulher, da Família e dos Direitos Humanos (MMFDH), entre os dias 1 e 25 de março ${ }^{11}$. O Fundo de População das Nações Unidas afirma que a permanência do isolamento por seis meses pode ser responsável por cerca de 31 milhões de casos extras de violência conjugal em todo o mundo ${ }^{12}$.

Frente a esse aumento das situações de violência, sobretudo àquelas em isolamento ou distanciamento social como forma de prevenir a transmissão pela Covid-19, faz-se necessário compreender quais motivos precipitam e/ou intensificam o fenômeno para que possam ser pensadas medidas de prevenção e enfrentamento da problemática. Considerando esse cenário no que diz respeito à violência conjugal, este estudo tem por objetivo: identificar elementos precipitadores/intensificadores da violência conjugal em tempos de pandemia da Covid-19.

\section{Metodologia}

Trata-se de uma revisão narrativa, utilizada para o alcance e a atualização do conhecimento sobre uma temática específica e pouco explorada ${ }^{13}$, possibilitando evidenciar novas ideias e assim ampliar o saber, como é o caso da violência conjugal no contexto da covid-19. O estudo partiu da seguinte questão orientadora: que elementos precipitam/intensificam a violência conjugal durante a pandemia do covid 19 ?

A busca na literatura ocorreu no mês de maio, na plataforma PubCovid-19, a qual está indexada na Biblioteca Nacional de Medicina dos Estados Unidos (PubMed) e no Banco de dados Excerpta Medica (EMBASE). Essa plataforma foi criada com o objetivo de compilar as publicações relacionadas à Covid-19 e organizar os artigos por áreas temáticas a fim de facilitar o acesso e direcionar o pesquisador. Para a realização da busca foram utilizados os seguintes descritores em inglês: "Domestic violente"; "Covid-19" e "Intimate Partner Violence". Foram incluídos artigos científicos que versavam sobre a Covid-19 e faziam interface com as temáticas: violência contra a mulher, violência conjugal e violência por parceiro íntimo. Excluíram-se artigos não disponíveis na integra de forma gratuita.

Foram selecionados inicialmente 13 artigos científicos. Após leitura dos títulos e resumos, 
quatro foram excluídos por não abordarem o tema de estudo, restando nove artigos para leitura na íntegra. Para melhor sistematização foi criado um instrumento para compilar as informações das publicações

Os estudos foram lidos exaustivamente, categorizados e analisados com vistas à busca dos elementos que precipitaram a violência no período da pandemia da COVID 19. A partir da exploração do material selecionado, foram elaboradas três categorias empíricas: Instabilidade econômica, Uso/abuso de álcool e outras drogas e Enfraquecimento da rede de apoio da mulher.

\section{Resultados}

Com base nas nove publicações selecionadas para este estudo foi desenvolvido um quadro (Quadro 1) expondo as características dessas publicações, segundo título, autoria, ano de publicação, país, periódico, metodologia adotada no estudo e elementos precipitadores/intensificadores de violência.

\section{Discussão}

De acordo com a literatura científica selecionada, um dos elementos precipitadores e/ou intensificadores da violência conjugal em tempos de pandemia da Covid-19 diz respeito à instabilidade econômica, expressa pela diminuição dos salários, desemprego, falta de recursos e dependência econômica feminina, o que predispõe a desentendimentos e consequentemente violência marital. $\mathrm{O}$ Fundo de População das Nações Unidas (UNFPA) publicou o documento intitulado "COVID-19 Um olhar para gênero: Proteção da Saúde e dos Direitos Sexuais e Reprodutivos e Promoção da Igualdade de Gênero", o qual aponta o aumento das tensões na família e o impacto econômico durante pandemia como fator que potencialmente poderia estar elevando a vulnerabilidade à violência doméstica, além de que os sistemas de proteção, neste período, podem enfraquecer ou serem interrompidos, devido à sobrecarga de cuidados em lidar com as vítimas do Covid-19 ${ }^{14}$.

O aumento do número de casos de violência conjugal sempre foi observado durante grandes catástrofes e crises econômicas, o que acaba sendo uma preocupação na atual conjuntura, principalmente pelo fato de não se ter uma previsão concreta de quando a pandemia vai acabar ${ }^{15}$. Esse contexto foi percebido, por exemplo, nos Estados Unidos, onde nove meses após o furacão Katrina, o terceiro mais mortífero do mundo, com efeitos econômicos de longo alcance, o número de violência por um parceiro triplicou e o estupro aumentou 16 vezes $^{16}$.

Esse aumento dos casos pode guardar relação com a falta de recursos econômicos e consequente recessão na economia ${ }^{17}$. A Organização Internacional do Trabalho prevê que, com a pandemia da Covid-19, cerca de 25 milhões de pessoas percam o seu emprego, repercutindo em grave recessão da economia mundial levando o estado ao pagamento de benefícios emergenciais à população desfavorecida ${ }^{18}$. A perda do emprego durante a pandemia e a consequente queda na renda familiar torna o espaço doméstico estressante, potencializando comportamentos violentos $^{19}$. Mesmo com as medidas adotadas para o pagamento de auxílio financeiro, o acesso a esses recursos está ocorrendo de forma demorada por conta da burocracia e da análise de informações, podendo acarretar em brigas familiares pela falta de recursos para a compra de alimentos.

Como na maioria das vezes a provisão dos recursos financeiros é de responsabilidade masculina, diante da ausência desses recursos, a esposa e os filhos tendem a realização de cobranças desse homem em relação à manutenção do sustento familiar. Todavia, essa atitude familiar pode catalisar conflitos. Essa situação é corroborada pela literatura selecionada quando afirmam que diante da carência de recursos financeiros por conta do desemprego existe uma limitação no acesso à alimentação e aos produtos de higiene, de modo que a exigência destes pelas mulheres leva muitos homens a agredirem suas companheiras ${ }^{20-24}$.

Outro elemento apontado enquanto precipitador e/ou intensificador da violência na conjugalidade guarda relação com o aumento do consumo de álcool e outras drogas. Sabe-se que estes fatores sempre estiveram correlacionados, independente da pandemia da Covid-19. Entretanto, observa-se um crescimento no uso dessas substâncias no ambiente doméstico por conta da pandemia e do isolamento social, repercutindo na elevação dos casos de violência marital no contexto do lar ${ }^{12,15,22,24,25}$.

A produção do saber já vem demonstrando que estar sobre o efeito do álcool e outras drogas potencializa comportamentos como euforia, autoconfiança e desinibição emocional podendo propiciar impulsos agressivos e perda de controle sobre o comportamento ${ }^{12,15}$. O uso/abuso de álcool e outras drogas no contexto do isolamento social guarda relação com hábitos anteriores à pandemia, associado à ansiedade relacionada à doença e em alguns casos pela falta de suporte de 
Quadro 1. Síntese dos estudos selecionados nas bases de dados PUBCOVID, Salvador, 2020.

\begin{tabular}{|c|c|c|c|c|}
\hline $\mathbf{N}^{\circ}$ & Título & $\begin{array}{l}\text { Autores, Ano, } \\
\text { Revista, País }\end{array}$ & Método & $\begin{array}{c}\text { Elementos precipitadores/ intensificadores } \\
\text { da violência }\end{array}$ \\
\hline 1 & $\begin{array}{l}\text { Increased Risk for Family } \\
\text { Violence During the } \\
\text { COVID-19 Pandemic }{ }^{26}\end{array}$ & $\begin{array}{l}\text { Kathryn LH et al. } \\
2020 \\
\text { Journal Pediatrics } \\
\text { Estados Unidos da } \\
\text { América (EUA) }\end{array}$ & $\begin{array}{l}\text { Revisão } \\
\text { narrativa }\end{array}$ & $\begin{array}{l}\text { - Elevação do consumo de substância psicoativas no } \\
\text { contexto doméstico está associado ao aumento de casos } \\
\text { de violência conjugal }\end{array}$ \\
\hline 2 & $\begin{array}{l}\text { Family violence and } \\
\text { COVID-19: Increased } \\
\text { vulnerability and reduced } \\
\text { options for support }^{22}\end{array}$ & $\begin{array}{l}\text { Usher K et al. } \\
2020 \\
\text { Int J Ment Health Nurs } \\
\text { Austrália }\end{array}$ & Reflexão & $\begin{array}{l}\text { - O isolamento social fez com que ocorresse um } \\
\text { enfraquecimento da rede de apoio à mulher em } \\
\text { situação de violência. } \\
\text { - A diminuição de recursos financeiros por conta do } \\
\text { desemprego } \\
\text { - O consumo elevado de álcool no ambiente doméstico. }\end{array}$ \\
\hline 3 & $\begin{array}{l}\text { The increase in domestic } \\
\text { violence during the social } \\
\text { isolation: what does it } \\
\text { reveals? }{ }^{23}\end{array}$ & $\begin{array}{l}\text { Vieira PR et al. } \\
2020 \\
\text { Rev Bras Epidemiol } \\
\text { Brasil }\end{array}$ & $\begin{array}{l}\text { Revisão } \\
\text { Sistemática }\end{array}$ & $\begin{array}{l}\text { - A convivência forçada pelo isolamento social } \\
\text { provocou o enfraquecimento da rede de apoio às } \\
\text { mulheres que já vivenciaram violência. } \\
\text { - Diminuição do salário e dependência financeira. }\end{array}$ \\
\hline 4 & $\begin{array}{l}\text { COVID-19: Reducing the risk } \\
\text { of infection might increase } \\
\text { the risk of intimate partner } \\
\text { violence }^{25}\end{array}$ & $\begin{array}{l}\text { Van Gelder N et al. } \\
2020 \\
\text { E Clinical Medicine } \\
\text { Holanda }\end{array}$ & Reflexão & $\begin{array}{l}\text { - Impacto financeiros na renda familiar provocados } \\
\text { pela diminuição dos salários. } \\
\text { - Consumo elevado de álcool e outras drogas. }\end{array}$ \\
\hline 5 & $\begin{array}{l}\text { The pandemic paradox: The } \\
\text { consequences of COVID-19 } \\
\text { on domestic violence }^{24}\end{array}$ & $\begin{array}{l}\text { Bradbury-Jones C, } \\
\text { Isham L } \\
2020 \\
\text { J Clin Nurs } \\
\text { Inglaterra }\end{array}$ & Reflexão & $\begin{array}{l}\text { - Cobrança feminina em relação ao sustento familiar. } \\
\text { - Consumo de álcool dentro de casa. }\end{array}$ \\
\hline 6 & $\begin{array}{l}\text { Health care practitioners' } \\
\text { responsibility to address } \\
\text { intimate partner violence } \\
\text { related to the COVID-19 } \\
\text { pandemic }^{15}\end{array}$ & $\begin{array}{l}\text { Bradley NL et al. } \\
2020 \\
\text { Can Med Assoc J } \\
\text { Canadá }\end{array}$ & $\begin{array}{l}\text { Revisão } \\
\text { Narrativa }\end{array}$ & $\begin{array}{l}\text { - Impacto financeiros na renda familiar provocados } \\
\text { pela diminuição dos salários. } \\
\text { - Enfraquecimento das redes de apoio à mulher. } \\
\text { - Uso/ abuso de substâncias alcoólicas. }\end{array}$ \\
\hline 7 & $\begin{array}{l}\text { Danger in danger: } \\
\text { Interpersonal violence during } \\
\text { COVID-19 quarantine }{ }^{20}\end{array}$ & $\begin{array}{l}\text { Mazza M et al. } \\
2020 \\
\text { Psychiatry Res } \\
\text { Itália }\end{array}$ & $\begin{array}{l}\text { Revisão } \\
\text { Narrativa }\end{array}$ & $\begin{array}{l}\text { - Instabilidade econômica, falta de recursos. } \\
\text { - Distanciamento de familiares e rede de apoio à } \\
\text { mulher. }\end{array}$ \\
\hline 8 & $\begin{array}{l}\text { Violence against women } \\
\text { during covid-19 pandemic } \\
\text { restrictions }{ }^{21}\end{array}$ & $\begin{array}{l}\text { Roesch E et al. } \\
2020 \\
\text { BMJ } \\
\text { Reino Unido }\end{array}$ & $\begin{array}{l}\text { Revisão } \\
\text { Narrativa }\end{array}$ & $\begin{array}{l}\text { - Enfraquecimento das redes de apoio à mulher. } \\
\text { - Restrição aos serviços de apoio psicossocial. } \\
\text { - Restrição ao uso das redes sociais pelo cônjuge. } \\
\text { - Impacto financeiros na renda familiar provocados } \\
\text { pelo desemprego }\end{array}$ \\
\hline 9 & $\begin{array}{l}\text { Covid-19: EU states report } \\
60 \% \text { rise in emergency calls } \\
\text { about domestic violence }\end{array}$ & $\begin{array}{l}\text { Mahase E } \\
2020 \\
\text { BMJ } \\
\text { Reino Unido }\end{array}$ & Reflexão & $\begin{array}{l}\text { - O uso/abuso de substâncias e alcoólicas e outras } \\
\text { drogas aumenta os casos de violência. }\end{array}$ \\
\hline
\end{tabular}

reuniões presenciais de grupos de apoio, a exemplo dos alcoólicos anônimos ${ }^{22,24,25}$.

Diante das situações supracitadas, associadas à ausência de grupos de apoio, o uso/abuso do álcool e outras substâncias pode ser compreendido como mecanismo de enfrentamento para o isolamento social. Todavia, este, por sua vez, favorece a desregulação das emoções e tende a desencadear comportamentos violentos ${ }^{22,25,26}$. Na Austrália, as medidas de distanciamento social foram implementadas com o fechamento de restaurantes e bares, levando a população ao consumo de álcool em casa, representando um aumento de mais de $36 \%$ da venda de bebidas alcoólicas na modalidade delivery, o que favoreceu elevação significativa nas denúncias por violência ${ }^{19}$. Diante disso, alguns países, a exemplo da Groenlândia, impuseram a proibição da venda de álcool na tentativa de conter casos de violência durante a quarentena ${ }^{27}$.

A carência de recursos financeiros associado ao uso/abuso de substâncias durante a pandemia que reverbera na violência marital faz com que 
os homens, como forma de se manter no poder em seus lares, limitem o acesso às redes de apoio da mulher, sejam estas informais, como amigos e familiares, ou formais, nos serviços jurídico-policiais e de saúde. Assim, no contexto da quarentena, as mulheres que são violentadas tendem a se sentirem ainda mais desassistidas, impossibilitadas de desabafar com alguém e de receber visitas ${ }^{15,22,23,25,26}$. Uma das formas de intensificar o mecanismo de coerção e enfraquecer às redes de apoio das mulheres na pandemia se dá por meio do controle dos meios de comunicação, a fim de evitar denúncias ou pedidos de ajuda ${ }^{21,22,24}$.

Outra situação que emergiu dos estudos remete a informações inverídicas fornecidas pelos cônjuges às mulheres com intuito de mitigar a busca por ajuda. Tal realidade foi evidenciada na Austrália, onde instituições de caridade a pessoas que sofrem violência doméstica receberam denúncias de mulheres cujos parceiros utilizavam informações incorretas sobre a extensão das medidas de quarentena ${ }^{22}$. Foi percebido também que as vítimas da violência conjugal podem ter receio de ir ao hospital por medo do descumprimento da quarentena e também de se contaminarem ${ }^{24}$.

Outro desafio encontrado faz referência a falhas no suporte a mulheres que vivenciam a violência no contexto de pandemia. Isso porque os profissionais de saúde enfrentam a necessidade de unir esforços para o cuidado relacionado às manifestações graves da Covid-19, fato este que desestruturou os demais atendimentos que não estão relacionados à doença ${ }^{12,15,21,26}$. Logo, à medida que os serviços de saúde se voltam ao cuidado a pessoas infectadas pela doença, as mulheres em vivência de violência perdem ainda mais espaço de atendimento nos serviços de saúde.

Diante o contexto apresentado pela literatura científica, urge que sejam ampliadas as redes de

\section{Colaboradores}

AF Silva, FM Estrela, CFS Soares, JRF Magalhães, NS Lima, AC Morais, NP Gomes e VLA Lima contribuíram igualmente nas etapas de concepção e projeto, redação do artigo e aprovação final da versão a ser publicada. apoio à mulher vítima de violência conjugal no contexto da pandemia da Covid-19. Estratégias exitosas foram criadas na Itália e no Canadá com o desenvolvimento de aplicativos que permitem que, em uma situação de perigo, as mulheres possam pedir ajuda sem precisar fazer uma ligação e uma campanha Signal for Help, na qual os profissionais de saúde eram informados do risco de violência por meio de um sinal manual durante a videoconferência da plataforma de telemedici$\mathrm{na}^{12,28}$. Tais estratégias são essenciais para se pensar a gestão para a prevenção e o enfrentamento da violência conjugal em tempos de pandemia.

\section{Considerações}

O estudo revela que os elementos precipitadores/ intensificadores de violência conjugal durante a pandemia da COVID 19 guardam relação com os aspectos socioeconômicos relacionados à redução dos salários, ao desemprego, à falta de recursos, à dependência econômica feminina e ao aumento do uso/abuso de substâncias pelos companheiros/cônjuges. Para além disso, outro elemento apontado pela literatura guarda relação com o enfraquecimento do suporte institucional e social às mulheres, onde inserem o apoio de instituições e de familiares.

É importante que nesse contexto sejam ampliadas as redes de apoio à mulher em situação de violência conjugal, com destaque para o uso de tecnologias digitais como possíveis ferramentas para triagem de casos de violência em tempos de pandemia. Acredita-se assim prevenir e enfrentar o fenômeno e consequentemente reduzir o alarmante número de casos, bem como mitigar as repercussões que esta acarreta para a vida de toda a família, sobretudo das mulheres.

\section{Referências}

1. World Health Organization (WHO). Coronavirus disease (COVID-19) outbreak [Internet]. Geneva: WHO; 2020. [acessado 2020 Mar 3]. Disponível em: https://www.who.int/emergencies/diseases/novel-coronavirus-2019

2. Croda JHR, Garcia LP. Resposta imediata da Vigilância em Saúde à epidemia da COVID-19. Epidemiol. Serv. Saúde 2020; 29(1). DOI: 10.5123/S167949742020000100021 
3. Brasil. Ministério da Saúde (MS). Coronavírus Brasil. Brasília: MS; 2020. [acessado 2020 Maio 15]. Disponível em: https://covid.saude.gov.br/

4. Nogueira L. Covid-19: Brasil se torna o $6^{\circ}$ país com mais casos; mortes superam 13 mil. 2020 [acessado 2020 Maio 14]. Disponível em: https://olhardigital. com.br/coronavirus/noticia/covid-19-brasil-setorna-o-7-pais-com-mais-casos-mortes-superam12-mil/98089

5. Brasil. Ministério da Saúde (MS). Boletim epidemiológico. Brasília: MS; 2020. [acessado 2020 Maio 12]. Disponível em: https://www.saude.gov.br/images/pdf /2020/April/06/2020-04-06-BE7-Boletim-Especialdo-COE-Atualizacao-da-Avaliacao-de-Risco.pdf

6. Rafael RMR, Mercedes Neto, Carvalho MMB, David HMSL, Acioli S, Faria MGA. Epidemiologia, políticas públicas e pandemia de Covid-19: o que esperar no Brasil? Revista Enfermagem UERJ 2020; 28:e49570.

7. Peterman A, Potts A, O'Donnell M, Thompson K, Shah N, Oertelt-Prigione S, van Gelder N. Pandemics and Violence Against Women and Children [Internet]. Center For Global Development; 2020 [acessado 12 Maio 2020]. Disponível em: https://www.cgdev.org/ sites/default/files/pandemics-and-violence-against-women-and-girls.pdf

8. Wanqing Z. Domestic Violence Cases Surger During COVID-19 Epidemic. Sixth Tone [Internet] 2020 [acessado 2020 Maio 12]. Disponível em: https:// www.sixthtone.com/news/1005253/domestic-violence-cases-surge-during-covid-19epidemic

9. La Provincia. Coronavirus: casi di violenza sulle donne raddopiatti in emergenza. La Provincia [Internet] 2020 [acessado 2020 Maio 12]. Disponível em: https:// www.laprovinciacr.it/news/italia-e-mondo/244892/ coronavirus-casi-di-violenza-sulle-donne-raddoppiati-in-emergenza.html

10. Euronews. Domestic violence cases jump 30\% during lockdown in France. Euronews [Internet] 2020 [acessado 2020 Maio 12]. Disponível em: https://www. euronews.com/2020/03/28/domestic-violence-casesjump-30-during-lockdown-in-france

11. Brasil. Coronavírus: sobe o número de ligações para canal de denúncia de violência doméstica na quarentena [Internet]. 2020 [acessado 2020 Maio 22]. Disponível em: https://www.gov.br/mdh/pt-br/assuntos/noticias /2020-2/marco/coronavirus-sobe-o-numero-de-liga coes-para-canal-de-denuncia-de-violencia-domestica-na-quarentena

12. Mahase E. Covid-19: EU states report $60 \%$ rise in emergency calls about domestic violence. BMJ 2020; 369:m1872

13. Toledo JA, Rodrigues MC. Teoria da mente em adultos: uma revisão narrativa da literatura. Bol. Acad. Paul. Psicol. [Internet], 2017 [acessado 2020 Maio 19]; 37(92):139-156. Disponível em: http://pepsic.bvsalud.org/scielo.php?script=sci_arttext\&pi$\mathrm{d}=\mathrm{S} 1415-711 \mathrm{X} 2017000100011 \& \operatorname{lng}=\mathrm{pt} \& \mathrm{nrm}=$ iso.

14. United Nations Population Fund HQ (UNFPA). Covid-19: resumo técnico proteção da saúde e dos direitos sexuais e reprodutivos e promoção da igualdade de gênero [Internet]. 2020 [acessado 2020 Maio 14]. Disponível em: https://brazil.unfpa.org/sites/default/ files/pub-pdf/covid19_olhar_genero.pdf
15. Bradley NL, DiPasquale AM, Dillabough K, Schneider PS. Health care practitioners' responsibility to address intimate partner violence related to the COVID-19 pandemic. Can Med Assoc J 2020; cmaj.200634.

16. Estadão São Paulo. Dois anos após o Katrina, New Orleans exige assistência [Internet]. 2007 [acessado 2020 Maio 05]. Disponível em: https://internacional. estadao.com.br/noticias/geral,dois-anos-apos-o-katrina-new-orleans-exige-assistencia, 43526

17. Saadat S, Rawtani D, Hussain CM. Environmental perspective of COVID-19. Sci Total Environ. 2020; 728: 138870. DOI: $10.1016 / j . s c i t o t e n v .2020 .138870$

18. Universidade Federal de Minas Gerais (UFMG). Estudo UFMG: violência doméstica pode ter aumentado com o isolamento social [Internet]. 2020 [acessado 2020 Maio 14]. Disponível em: https://ufmg.br/comunicacao/assessoria-de-imprensa/release/estudo-ufmg-violencia-domestica-pode-ter-aumentado-com -o-isolamento-social

19. Commonwealth Bank Group. An early look at how the Coronavirus is affecting household spending [Internet] Commonwealth Bank Group: Austrália. 2020 [acessado 2020 abr 10]. Available from: https://www. commbank.com.au/guidance/business/an-early-look -at-how-coronavirus-is-affecting-household-spending-202004.html

20. Mazza M, Marano G, Lai C, Janiri L, Sani G. Danger in danger: Interpersonal violence during COVID-19 quarantine. Psychiatry Res 2020; 289:113046.

21. Roesch E, Amin A, Gupta J, García-Moreno C. Violence against women during covid-19 pandemic restrictions. BMJ 2020; 1712 .

22. Usher K, Bhullar N, Durkin J, Gyamfi N, Jackson D. Family violence and COVID-19: Increased vulnerability and reduced options for support. Int J Ment Health Nurs 2020; inm.12735.

23. Vieira PR, Garcia LP, Maciel ELN. Isolamento social e o aumento da violência doméstica: o que isso nos revela? Rev Bras Epidemiol. 2020; 23.

24. Bradbury-Jones C, Isham L. The pandemic paradox: The consequences of COVID-19 on domestic violence. J Clin Nurs 2020; jocn.15296.

25. Van Gelder N, Peterman A, Potts A, O’Donnell M, Thompson K, Shah N, Oertelt-Prigione S; Gender and COVID-19 working group. COVID-19: Reducing the risk of infection might increase the risk of intimate partner violence. E Clinical Medicine 2020; 21:100348.

26. Kathryn LH, Myo TM, Charles HZ. Increased risk for family violence during the COVID-19 pandemic. $P e$ diatrics 2020; e20200982.

27. Scandinavian Way. Groenlândia impõe restrição extra na quarentena: a de bebidas [Internet]. 2020 [acessado 2020 Maio 22]. Disponível em: https://scandinavianway.com.br/groenlandia-proibe-quarentena-bebidas-alcoolicas/

28. Higgins N. Coronavirus: when home gets violent under lockdown in Europe [Internet]. BBC News 2020 [acessado 2020 abr 27]. Available: www.bbc.com/ news/world-europe -52216966

Artigo apresentado em 22/05/2020

Aprovado em 24/05/2020

Versão final apresentada em 26/05/2020 